\title{
Gliosarcoma, una variante del glioblastoma primario IDH-wildtype: serie de tres casos y revisión de la literatura médica
}

\author{
José Alfredo Espinosa-Mora', Luis Eduardo Moreira-Ponce1, Carlos Alberto Murillo-Ponce, \\ Nataly Mireya Alvear-Quito', Christian Emmanuel Cardona-Mejia', Arturo Ayala-Arcipreste1, \\ Paola Yolotzin Valenzuela-Torres², Teresa Cuesta-Mejías², Ricardo Ramírez-Aguilar ${ }^{3}$ y Rafael Mendizábal-Guerra ${ }^{4}$
}

${ }^{1}$ Servicio de Neurocirugia Hospital Juárez de México; ${ }^{2}$ Servicio de Anatomia Patologica Hospital Juárez de México; ${ }^{3}$ Servicio de Neurocirugia Hospital General Balbuena; ${ }^{4}$ Jefe de Servicio de Neurocirugía Hospital Juárez de México. Ciudad de México, México.

\begin{abstract}
Resumen
Introducción: El gliosarcoma es una lesión primaria del sistema nervioso central infrecuente, considerada de grado IV por la Organización Mundial de la Salud (OMS) dentro de la nueva clasificación de 2016. Es una variante del glioblastoma (GBM) descrita como IDH-wildtype y se caracteriza por presentar un patrón bifásico: un componente glial y otro mesenquimatoso. Las similitudes clínicas del gliosarcoma con el GBM han llevado a muchos autores a concluir que son clínicamente indistinguibles; sin embargo, hay una serie de características importantes y distintas del gliosarcoma que sugieren que es una entidad separada. Los rasgos más llamativos para diferenciar ambas enfermedades incluyen ubicación y características imagenológicas específicas, pero el diagnóstico definitivo se realiza por estudio histopatológico y se confirma con pruebas moleculares. Caso 1: Mujer de 51 años con cuadro de dos meses de cefalea holocraneal -que aumenta con maniobras de Valsalva y mejora con antiinflamatorios no esteroideos (AINE)-, fosfenos, náuseas y vómitos posprandiales. En la exploración neurológica se detecta: papila con edema, hemianopsia homónima derecha, hemiparesia e hiperreflexia izquierdas, y signo de Babiński izquierdo. Caso 2: Mujer de 40 años, con cefalea frontal vespertina, labilidad emocional, disfasia, náuseas, vómitos y disminución de la agudeza visual. En la exploración neurológica se observa: disfasia motora, edema de papila, agudeza visual 20/60 bilateral, hemiparesia derecha, apatía y alteración en la planeación. Caso 3: Varón de 58 años con cuadro de cinco semanas de cefalea progresiva, frontal y vespertina, y disminución de la fuerza en los miembros pélvicos simétricos. En la exploración neurológica se encuentra: agudeza visual 20/40 bilateral, hemiparesia izquierda, hiperreflexia y signo de Babiński izquierdos. Los tres pacientes se sometieron a craneotomía y resección total de la lesión, en la que se encontró una lesión sólida de consistencia ahulada con gliosis perilesional. El estudio histopatológico reportó gliosarcoma en los tres casos. Conclusiones: Presentamos esta serie de casos con diagnóstico de gliosarcoma por su escasa frecuencia; además, realizamos una revisión de la literatura médica.
\end{abstract}

Palabras clave: Gliosarcoma. Glioblastoma. Tumor mesenquimatoso. Sarcoma.

\section{Correspondencia:}

José Alfredo Espinosa-Mora

Hospital Juárez de México

Av. Instituto Politécnico Nacional, 5160

Col. Magdalena de las Salinas,

Del. Gustavo A. Madero

Fecha de recepción: 25/05/2017

Fecha de aceptación: 28/06/2017

C.P. 07760, Ciudad de México, México

E-mail: al.campa11@ hotmail.com

1665-5044/@ 2018. Academia Mexicana de Neurología A.C. Publicado por Permanyer México. Este es un artículo Open Access bajo la licencia CC BY-NC-ND (http://creativecommons.org/licenses/by-nc-nd/4.0/).

Disponible en internet: 13-11-2018 Rev Mex Neuroci. 2018;19(5):47-53 www.revmexneuroci.com 
Rev Mex Neuroci. 2018;19

\title{
Gliosarcoma, a variant of the primary Glioblastoma IDH-Wildtype: series of three cases and literature review
}

\begin{abstract}
Introduction: Gliosarcoma is an uncommon primary central nervous system lesion; considered as WHO Grade IV, within the new classification of 2016 is a variant of Glioblastoma (GBM) described as IDH-wildtype, is characterized by a biphasic pattern; with a glial and a mesenchymal component. Clinical similarities to GBM have led many authors to conclude that they are clinically indistinguishable; however, there are a number of important and distinct features of Gliosarcoma that suggest that it is a separate entity. The most striking features to differentiate them include location, differential imaging aspect and gross appearance, but the definitive diagnosis is by histopathological study and confirmed with molecular tests. Case 1:51-yearold female with a 2-month history of holocranial headache, increased with valsalva maneuvers, improvement with NSAIDs, phosphenes, nausea and postprandial vomiting. Neurological examination: papilloedema, right homonymous hemianopsia, left hemiparesis and hyperreflexia, left Babinsky. Case 2: 40-year-old female, with frontal, evening headache, emotional lability, dysphasia, nausea, vomiting and decreased vision. Neurological examination: motor dysphasia, papillodema, decreased vision, right hemiparesis, apathy, alteration in planning. Case 3: Male, 58-year-old, with a 5-week history of progressive headache, frontal, decreased symmetrical pelvic limb strength. Neurological examination: decreased visión bilateral, left hemiparesis, hypereflexia and left Babinsky. The three patients underwent craniotomy and total resection of the lesion, where a solid lesion of "smoothed"consistency with perilesional gliosi, the histopathological study reports gliosarcoma in all. Conclusions: We present this series of cases with diagnosis of gliosarcoma, which due to the infrequent frequency, we review the literature and present them.
\end{abstract}

Key words: Gliosarcoma. Glioblastoma. Mesenchymal tumor. Sarcoma.

\section{Introducción}

El gliosarcoma es una lesión primaria del sistema nervioso central poco frecuente. Fue descrita por primera vez por $\mathrm{H}$. Stroebe en $1895^{1,2}$ y es considerada un tumor de grado IV por la OMS. Es una variante del GBM y se caracteriza por presentar un patrón bifásico con dos componentes tisulares: uno de origen glial y otro mesenquimatoso $0^{1,3,4-8}$. Dentro de la nueva clasificación de la OMS de 2016, se encuentra dentro de la variante del GBM IDH-wildtype, junto con el GBM de células gigantes y el GBM epiteloide, considerados variantes primarios ${ }^{4,5}$.

Es más frecuente en el sexo masculino, con 1.8-3:1, y la localización más común es en el lóbulo temporal ${ }^{3,6}$.

Las similitudes clínicas con otras variantes del GBM han llevado a muchos autores a concluir que estos tumores son clínicamente indistinguibles; sin embargo, hay una serie de características importantes y distintas que sugieren que el gliosarcoma es una entidad separada. Los rasgos más llamativos para diferenciarlos incluyen su ubicación y sus características específicas por imagen ${ }^{8}$, pero el diagnóstico definitivo se realiza por estudio histopatológico y es confirmado por estudios moleculares ${ }^{4,6}$.

Son pocas las series de casos reportados en la literatura médica y con un limitado número de pacientes por serie, debido a la poca frecuencia de esta entidad. Nosotros presentamos esta serie de tres casos que tuvimos durante un periodo de cinco años aproximadamente.

\section{Reporte de casos}

\section{Caso 1}

Mujer de 51 años, sin ningún antecedente de importancia, que presentaba cuadro clínico de dos meses de evolución con cefalea holocraneal opresiva, de intensidad 9/10 EVA, que aumentaba por las noches y con maniobras de Valsalva y mejoraba con AINE parcialmente, acompañada de fosfenos, náuseas y vómitos posprandiales. En la exploración neurológica presentó Glasgow de 15, papila con bordes borrados, pulso venoso disminuido, hemianopsia homónima derecha, pupilas isocóricas de $3 \mathrm{~mm}$ con respuesta a la luz, resto de nervios craneales sin alteración, hemiparesia izquierda $3 / 5$, hiperreflexia izquierda sin clono, signo de Babiński izquierdo, sensibilidad, y cerebelo y meníngeos sin alteraciones.

Se realizó RMN de encéfalo, en la que se observó lesión corticosubcortical parietooccipital derecha de bordes definidos adyacente a la hoz cerebral, multilobulada y que realzaba con gadolinio de forma heterogénea, con áreas hipointensas en el centro y un edema digitiforme perilesional, con desplazamiento de línea media de $1 \mathrm{~cm}$ y colapso de ventrículo lateral ipsilateral 

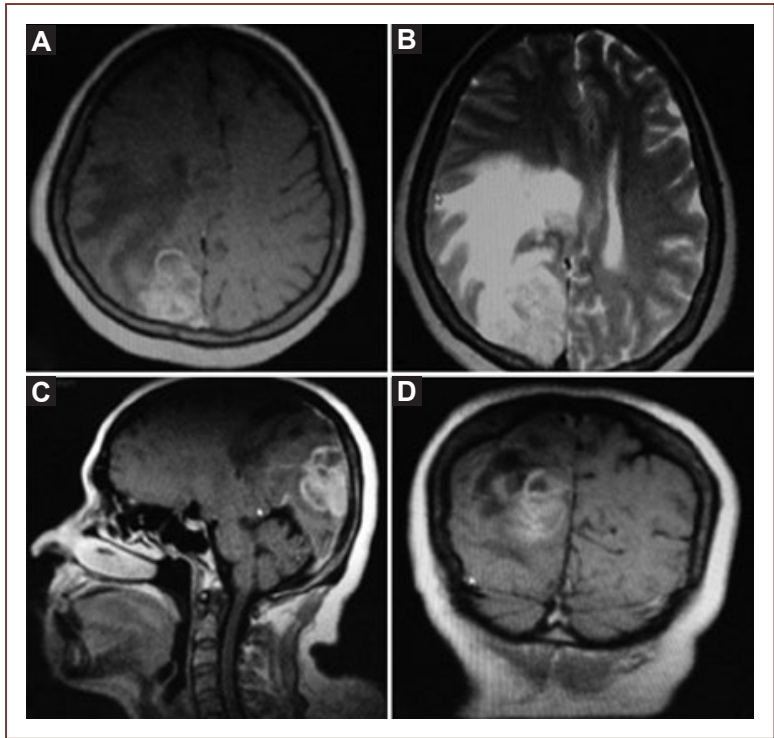

Figura 1. RMN contrastada. Se observa lesión corticosubcortical parietooccipital derecha de bordes definidos adyacente a la hoz cerebral, multilobulada, que realza con gadolinio de forma heterogénea, con áreas hipointensas en el centro, eje axial contrastado (A), axial T2 donde se visualiza el importante edema digitiforme perilesional (B), eje sagital contrastado (C) y eje coronal contrastado (D).

(Fig. 1). La paciente fue sometida a cirugía, en la que se encontró lesión adherida a hoz de cerebro de consistencia ahulada y con bordes irregulares.

El estudio histopatológico reportó neoplasia de alto grado, pleomórfica, con metaplasia mesenquimatosa y con componentes gliales con GFAP positivas. Presentó vimentina, desmina y Ki-67 positivos

\section{Caso 2}

Mujer de 40 años que presentaba cuadro de cefalea frontal, de predominio vespertino, con labilidad emocional y dificultad para la emisión del lenguaje, náuseas, vómitos y disminución de la agudeza visual. En la exploración neurológica se observó Glasgow de 15, disfasia motora, edema de papila, agudeza visual 20/60 bilateral, hemiparesia derecha 4+/5, apatía, falta de fluidez verbal, déficit de planeación y datos clínicos en relación con síndrome frontal dorsolateral.

Se realizó TAC craneal, en la que se detectó una lesión hiperdensa en la región frontal izquierda, de bordes irregulares heterogéneos, que cruza la línea media e invade el hemisferio contralateral y edema perilesional.
La paciente fue sometida a cirugía, en la que se encontró lesión frontal izquierda adherida al tercio anterior del seno sagital, de consistencia ahulada, vascularizada, con áreas adheridas a tejido sano, alternando con áreas con plano de disección bien delimitado de tejido sano.

El estudio histopatológico reportó neoplasia de alto grado, epiteloide y fusocelular, con metaplasia mesenquimatosa, positiva en inmunomarcación para proteína ácida gliofibrilar, vimentina, S-100 y actina de músculo liso.

\section{Caso 3}

Varón de 58 años de edad sin antecedentes de importancia que presentaba cuadro de cinco semanas de evolución caracterizado por cefalea progresiva pulsátil, de predominio frontal y vespertina; posteriormente presentó disminución de la fuerza de ambos miembros pélvicos de forma simétrica. En la exploración neurológica se observó Glasgow de 15, funciones mentales superiores normales, fondo de ojo sin edema de papila, agudeza visual $20 / 40$ bilateral, hemiparesia izquierda $4 / 5$, con hiperreflexia sin clono y signo de Babiński izquierdo.

Se realizó RMN, que mostró una lesión heterogénea en el lóbulo frontal derecho, hiperintensa en T2 y FLAIR, con edema digitiforme perilesional y desviación de línea media (Fig. 2).

El paciente fue sometido a manejo quirúrgico, en el que se encontró lesión frontal derecha interhemisférica indurada, sólida, de consistencia ahulada y con gliosis perilesional (Fig. 3).

El estudio histopatológico mostró una densa proliferación tumoral con patrón bifásico, alternando un componente glial y uno sarcomatoide. El componente glial se caracterizaba por células gemistocíticas, pleomórficas, con alto índice mitósico y proliferación endovascular, mientras que en el componente sarcomatoide las células mesenquimales eran fusiformes con atipia nuclear y mitosis, algunas con diferenciación miofibroblástica alrededor de las paredes gruesas de los vasos sanguíneos.

Las áreas gliales fueron GFAP positivas y las áreas mesenquimales ricas en retículo, actina y GFAP negativas. El resto de la inmunomarcación realizada fue vimentina, desmina positivas y $\mathrm{Ki}-67$ del $40 \%$ en ambos componentes, CD34 positiva en vasos (Figs. 4 y 5).

\section{Discusión}

El gliosarcoma es una lesión poco frecuente, con una incidencia del $1.8-2.8 \%$ de los GBM${ }^{1}$. Predomina en la 


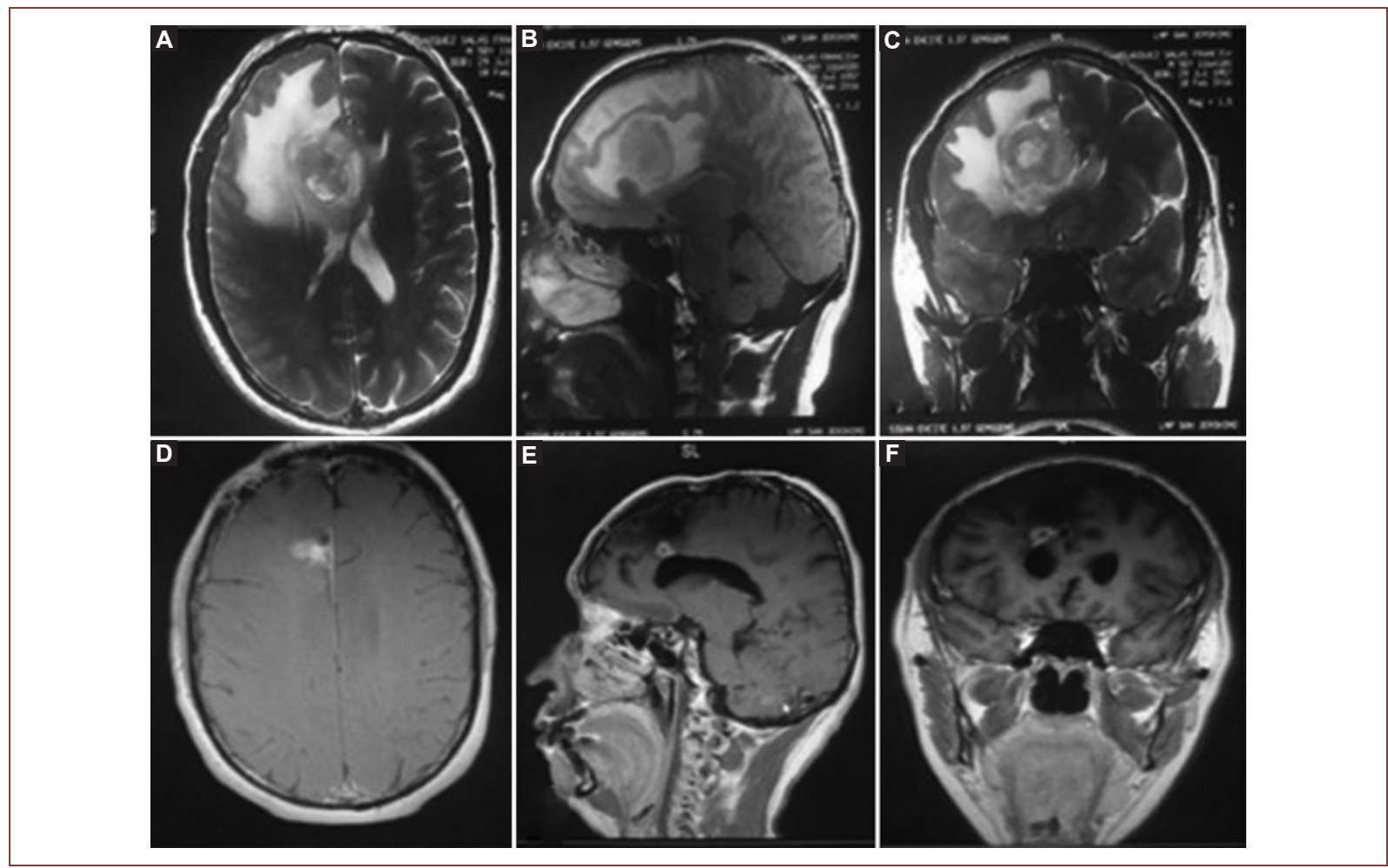

Figura 2. A, B y C: RMN preoperatoria ponderación T2, donde se observa lesión heterogénea hiperintensa, con bordes definidos y edema digitiforme. D, E y F: RMN postoperatoria, en la que se observa el trayecto de la brecha quirúrgica y los cambios posquirúrgicos, así como la lesión residual mínima, a cuatro meses de la cirugía, en tratamiento bajo radioterapia.

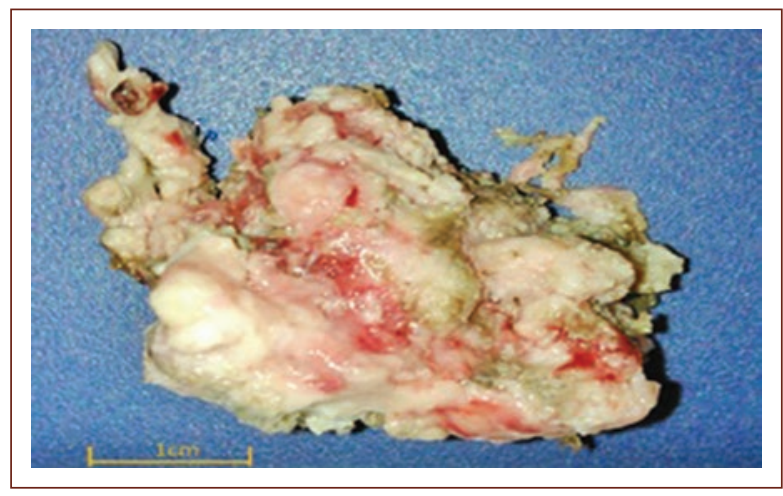

Figura 3. Pieza macroscópica de tumor en región frontal derecha.

población masculina, con 1.8-3:12-8, con una edad promedio entre los 40 y 60 años, aunque también se han reportado casos en edad pediátrica ${ }^{1}$, con características clínicas y radiológicas similar a éste ${ }^{7}$. La localización más frecuente es supratentorial, especialmente en la región periférica del lóbulo temporal, y por orden de frecuencia le siguen: lóbulo frontal, parietal, occipital y cuerpo calloso. Sólo se han reportado tres casos de gliosarcoma infratentorial ${ }^{3,6}$, algunos autores reportaron escasos casos intraventriculares y Doddamani, et. al. reportaron un único caso en localización intraventricular pero con aspecto quístico².

El primero que describió esta lesión fue H. Stroebe en 1895, quien usó el término gliosarcoma para referirse a un tumor formado por una combinación de GBM y sarcoma ${ }^{8,9}$. El término no ganó amplia aceptación hasta 1955, cuando Feigen y Cross describieron en detalle tres pacientes con este tumor ${ }^{10}$.

Existen múltiples hipótesis en relación con el origen de esta neoplasia, aunque mayoritariamente se cree que el tumor puede ser producto de un GBM con cambio metaplásico; otras hipótesis sugieren una transformación maligna de las células gliales que rodean un sarcoma preexistente o son simplemente la coexistencia de dos tumores en crecimiento ${ }^{11}$. Recientes estudios genéticos han demostrado un origen único (glial) de ambos componentes tumorales, encontrándose en dos casos idénticas mutaciones del gen PTEN en los componentes glial y sarcomatoso. Estas muestras presentaban deleción 


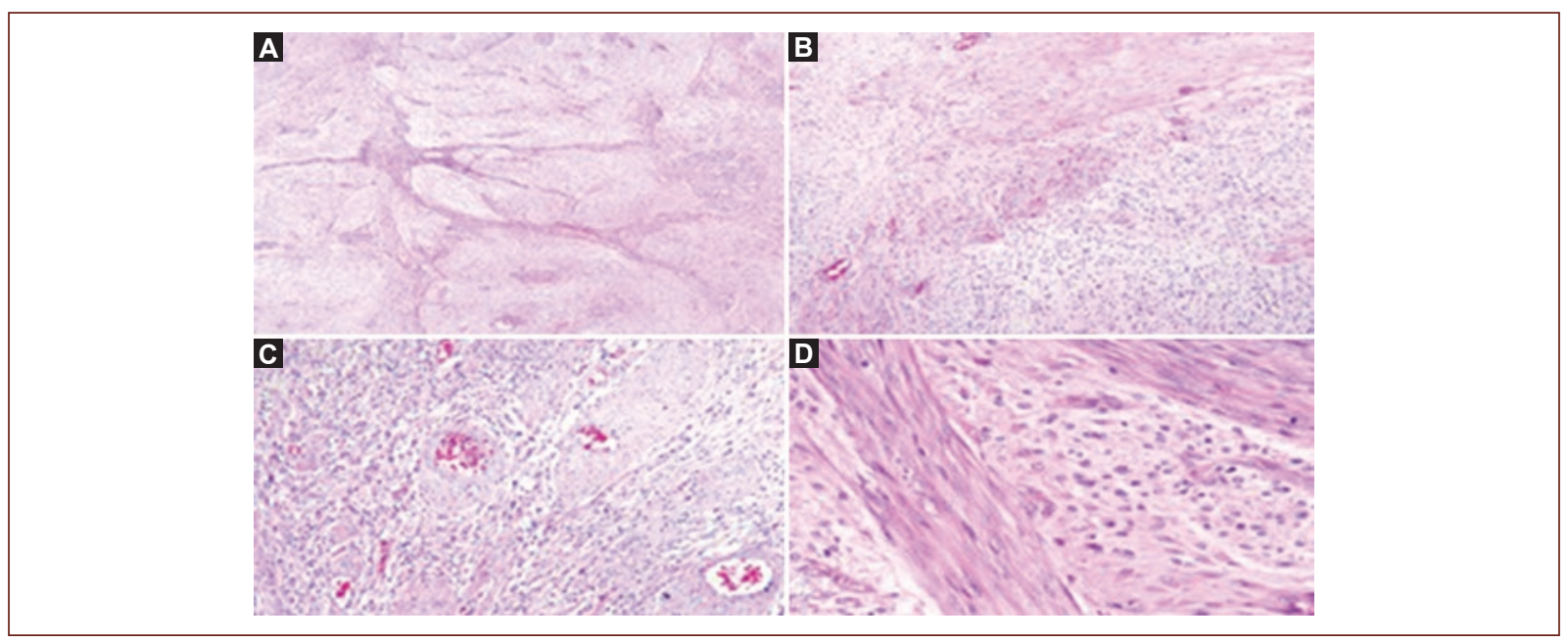

Figura 4. Cortes teñidos con H\&E. A: proliferación tumoral con patrón bifásico. B y C: componente glial (células gemistiocíticas, pleomórficas y proliferación endovascular) y componente sarcomatoide (células mesenquimales fusiformes alrededor de las paredes gruesas de los vasos sanguíneos). D: atipia citológica y mitosis.

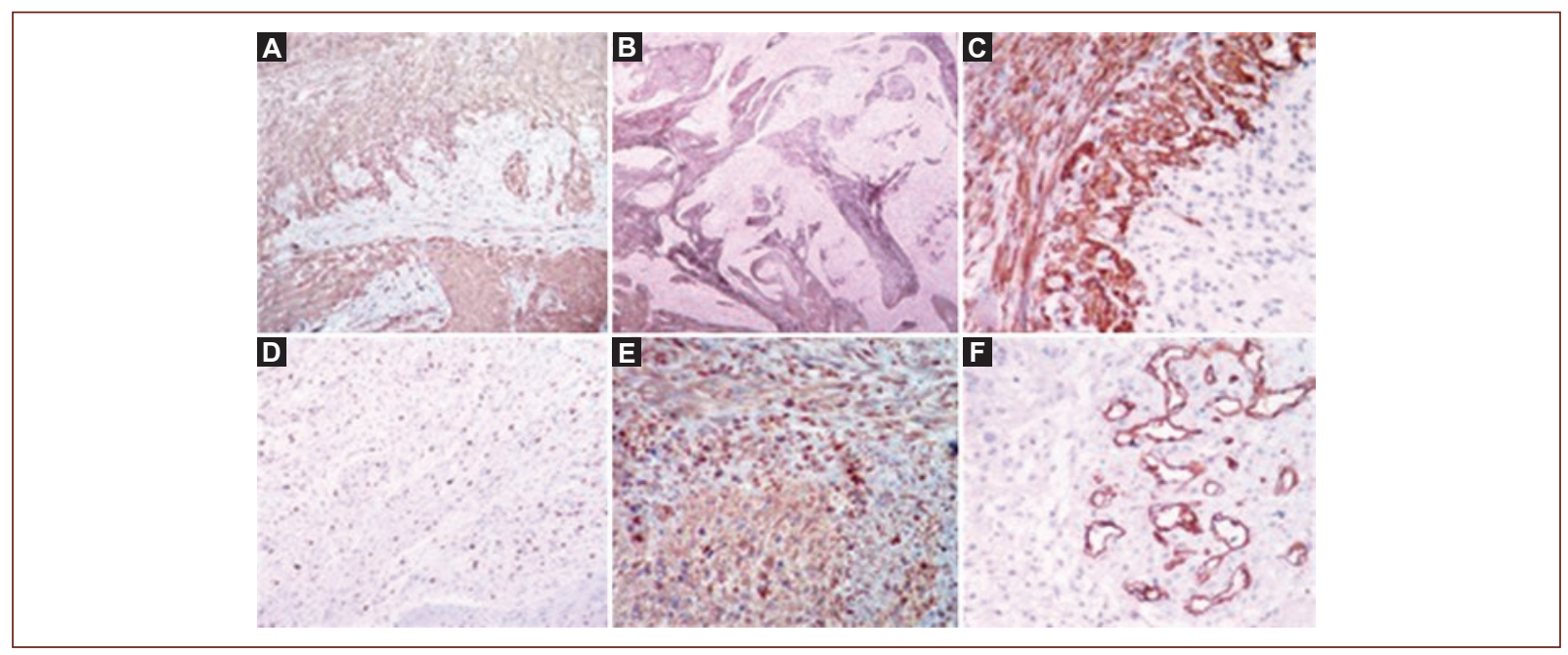

Figura 5. Inmunohistoquímica: componente glial positivo a GFAP (A: GFAP 40×), componente mesenquimal rico en retículo (B: retículo 10x), el cual muestra diferenciación miofibloblástica, con actina positiva (C: actina 40×), ambos componentes con proliferación celular con un Ki-67 del 40\% (D: Ki-67 40x) y positividad de vimentina (E: vimentina 40×). CD34 positivo únicamente en vasos (F: CD34 40x).

del p16, idéntica mutación del p53 o coamplificación de MDM2 y CDK4 en ambas áreas tumorales. La presencia de idénticas alteraciones genéticas en ambos componentes, glioblastomatoso y sarcomatoso, apoyan manifiestamente un origen único del gliosarcoma ${ }^{3,9}$.

Los signos y síntomas reportados son consistentes con los de un tumor intracraneal en rápida expansión, con aumento de la presión intracraneal, incluyendo afasia, cefalea, hemiparesia, convulsiones y deterioro cognitivo, dependiendo de la ubicación de la lesión 8-10. El gliosarcoma es indistinguible del GBM clínicamente; sin embargo, algunas características específicas despiertan la sospecha de esta lesión, tales como los hallazgos imagenológicos característicos, por lo que se debe tener en consideración tras un cuadro clínico de corta duración, en un paciente joven y con estudios de imagen con sospecha de GBM pero no concluyentes con éste ${ }^{6-9}$. 
Los hallazgos radiológicos del gliosarcoma son variables. En la TC son similares a los de un GBM infiltrante, apareciendo como una lesión hiperdensa bien definida con realce heterogéneo o en anillo debido a un componente fibroso; un GBM generalmente muestra bajo 0 intermedio realce ${ }^{8}$. En un estudio, Zhang, et al. informaron que los gliosarcomas se caracterizan por ser masas irregulares con bordes bien definidos (tanto en la TC como en las resonancias magnéticas), con una pared externa lisa bien delimitada, independientemente de edema periférico, que le confiere ciertas características parecidas a un meningioma ${ }^{11}$. En nuestros casos, como en los de la literatura médica, los estudios de imagen mostraron lesiones hiperdensas con realce heterogéneo con el medio de contraste, pero con un borde bien definido, tanto que incluso en uno de ellos se tuvo la sospecha de meningioma atípico.

En el corte histológico, una mezcla de tejido glioblastomatoso y sarcomatoide le confieren un llamativo patrón bifásico de tejido. La porción glial es de origen astrocítico -mostrando sobre todo las características típicas de un GBM-, en el cual se puede presentar una diferenciación epitelial, manifiestas características carcinomatosas con formaciones similares a glándulas adenoides y metaplasia escamosa. El componente sarcomatoso por definición muestra signos de la transformación maligna, tales como atipia nuclear, actividad mitótica y necrosis, y a menudo demuestra el patrón típico de fibrosarcoma, con largos haces densos de células fusiformes. Un porcentaje de casos pueden mostrar líneas adicionales de diferenciación mesenquimal como formación de cartílago, hueso, tejido osteoidecondral, músculo liso y estriado e incluso características lipomatosas ${ }^{12,13-15}$. La distinción entre los dos componentes es facilitada por el uso combinado de histoquímica y tinción inmunohistoquímica. Las partes mesenquimales pueden ser teñidas con retículo y/o Masson. Este componente no expresa GFAP, que, por el contrario, se observa en la parte glial'12,15.

El tratamiento es multidisciplinario, e incluye resección quirúrgica máxima y radioterapia y quimioterapia postoperatorias ${ }^{11}$, aunque el pronóstico es pobre a pesar de estas intervenciones. Algunos autores refieren que el gliosarcoma presenta la menor tasa de sobrevida de todos los GBM, presentando un máximo de 4-6 me$\mathrm{ses}^{8,10}$; sin embargo, otros autores han sugerido que tienen una evolución más favorable que otras variantes del GBM, aunque grandes ensayos clínicos no han mostrado diferencias significativas en ese sentido ${ }^{11}$. Existen reportes de casos con prolongada supervivencia, de
130 y 48 meses, que se manejaron con resección total, radioterapia y quimioterapia, en los cuales se menciona que el componente fibroso limita el crecimiento y le da una cápsula que permite una resecabilidad total ${ }^{17}$. Gran parte de la literatura médica concuerda en que el gliosarcoma tiene una propensión a la metástasis a distancia, y los sitios de mayor afección reportados son pulmón, hígado y huesos ${ }^{17}$.

En nuestra institución, los tres casos presentados fueron sometidos a cirugía radical, en los que se reportó la característica macroscópica descrita como de consistencia ahulada durante el procedimiento, además de visualizarse los bordes definidos y alta vascularidad. El diagnóstico definitivo fue mediante histopatología en los tres casos, con reporte del patrón bifásico característico, con metaplasia mesenquimatosa, además de la inmunomarcación positiva para vimentina, S-100, proteína ácida gliofibrilar, al igual que en la literatura médica; sin embargo, en ninguno de los tres casos se realizaron estudios moleculares en relación con la IDH para descartar mutaciones.

El seguimiento de los tres pacientes fue multidisciplinario, junto con el Servicio de Radiooncología y manejados con radioterapia. Hasta el momento los tres pacientes siguen con vida, aún sin concluir su tratamiento. Clínicamente presentan buena respuesta hasta el momento, pero uno de los casos presentó recidiva en un periodo de seguimiento de 12 meses y requirió una segunda intervención quirúrgica.

\section{Conclusiones}

Con esta serie de casos presentados nos percatamos de que la población afectada pertenece principalmente a la cuarta-sexta década de la vida y que, a pesar del manejo multidisciplinario, los resultados obtenidos no son los mejores. Por otra parte, nos dimos cuenta de que los estudios de imagen difieren un tanto de las otras variantes del GBM y tienen ciertas similitudes con un meningioma, pero el cuadro clínico de rápida progresión nos ayuda a diferenciarlo de éste. A pesar de ello, se requieren estudios más amplios, con mayor número de casos y con un seguimiento prolongado para evaluar el pronóstico en comparación con otras variantes del GBM, así como estudios moleculares para descartar mutaciones del IDH.

\section{Bibliografía} 1. Mena US, Sharma S, Chopra S, Jain SK. Gliosarcoma: a rare variant of
glioblastoma multiforme in paediatric patient: case report and review of
literatura. World J Clin Cases. 2016;4(9):302-5. 
2. Doddamani RS, Meena R, Mohan MS, Venkataramanaa NK, Tophkhane M, Garg SK. Intraventricular gliosarcomas: literature review and a case description. World Neurosurg. 2016;90:707.e5-707.e12.

3. Louis DN, Ohgaki H, Wiestler OD, Cavenne WK. Gliosarcoma. En: WHO classification of tumours of the central neurvious system. $4 .^{\mathrm{a}}$ ed. Lyon: IARC; 2007. p. 48-9.

4. Louis DN, Perry A, Rifenberger G, von Demling A, Figarella-Branger D Cavenee WK, et al. The 2016 World Healt Organization Classification of Tumors of the Central Nervous System: a summary. Acta Neuropathol. 2016;131(6):803-20.

5. Oh JE, Ohta T, Nonoguchi N, Satomi K, Capper D, Pierscianek D, et al. Genetic alterations in gliosarcoma and giant cell glioblastoma. Brain Pathol. 2016;26(4):517-22.

6. Moon SK, Kim EJ, Choi WS, Ryu CW, Park BJ, Lee J. Gliosarcoma of the cerebellar hemisphere: a case report and review of the literature. Korean J Radiol. 2010;11(5):566-70.

7. Delgado-Hernández $\mathrm{C}$, et al. Gliosarcoma de lóbulo parietal: reporte de un caso y revisión de la literatura. Rev Hosp Jua Mex. 2008; 75(1):73-7

8. Han SJ, Yang I, Tihan T, Prados MD, Parsa AT. Primary gliosarcoma: key clinical and pathologic distinctions from glioblastoma with implications as a unique oncologic entity. J Neurooncol. 2010;96(3):313-20.
9. Atienza-Cuevas L, Añón-Requena MJ, Guerrero-Cauqui R, Beltrán-Ruiz HM, Pérez-Requena J. Gliosarcoma con diferenciación adenoide y fibras de Rosenthal. Presentación de un caso. Rev Esp Patol. 2006;39(3):193-6.

10. Kozak KR, Mahadevan A, Moody JS. Adult gliosarcoma: epidemiology, natural history, and factors associated with outcome. Neuro Oncol. 2009;11(2):183-91.

11. Romero-Rojas AE, Díaz-Pérez JA, Ariza-Serrano LM, Amaro D, Lozano-Castillo A. Primary gliosarcoma of the brain: radiologic and histopathologic features. Neuroradiol J. 2013;26(6):639-48.

12. Perry A, Brat DJ. Practical surgical neuropathology a diagnostic approach. EE.UU.: Churchill Livingstone; 2010. p. 70-2, 75, 76

13. Prayson R, Kleinschmidt-DeMasters B, Cohen ML. Brain tumors. EE.UU: Demos Medical; 2010. p. 34-6.

14. Love S, Louis D, Ellison D. Greenfield's neuropathology. London: Holdder A, editor. 8. ${ }^{\mathrm{a}}$ ed. p. 1854

15. Kumar P, Singh S, Kumar P, Krishnani N, Datta NR. Gliosarcoma: an audit from a single institution in India of 24 post-irradiated cases over 15 years. J Cancer Res Ther. 2008;4(4):164-8.

16. Huo Z, Yang D, Shen J, Li Y, Wu H, Meng Y, et al. Primary gliosarcoma with long-survival: report of two cases and review of literature. Int J Clin Exp Pathol. 2014;7(9):6323-32.

17. Rizvi S, Asghar AH, Mehboob J. Gliosarcoma: a rare variant of glioblastoma multiforme. J Pack Med Assoc. 2010;60(9):773-5. 